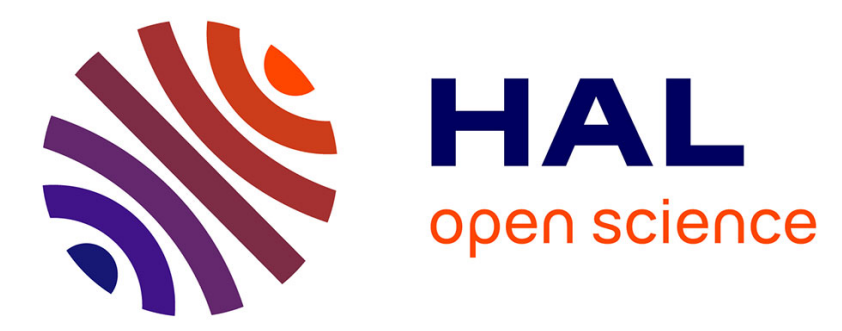

\title{
Systems Biology Approach and Mathematical Modeling for Analyzing Phase-Space Switch During Epithelial-Mesenchymal Transition
}

Chiara Simeoni, Simona Dinicola, Alessandra Cucina, Corrado Mascia, Mariano Bizzarri

\section{To cite this version:}

Chiara Simeoni, Simona Dinicola, Alessandra Cucina, Corrado Mascia, Mariano Bizzarri. Systems Biology Approach and Mathematical Modeling for Analyzing Phase-Space Switch During EpithelialMesenchymal Transition. M. Bizzarri (ed.). Systems Biology, 1702, pp.95-123, 2018, Methods in Molecular Biology, 10.1007/978-1-4939-7456-6_7 . hal-02520739

\section{HAL Id: hal-02520739 \\ https://hal.science/hal-02520739}

Submitted on 26 Mar 2020

HAL is a multi-disciplinary open access archive for the deposit and dissemination of scientific research documents, whether they are published or not. The documents may come from teaching and research institutions in France or abroad, or from public or private research centers.
L'archive ouverte pluridisciplinaire HAL, est destinée au dépôt et à la diffusion de documents scientifiques de niveau recherche, publiés ou non, émanant des établissements d'enseignement et de recherche français ou étrangers, des laboratoires publics ou privés. 


\title{
Systems Biology approach and Mathematical Modeling for analyzing phase-space switch during Epithelial-Mesenchymal Transition
}

RUNNING TITLE: mathematical modeling of phase transitions in biology

\author{
Chiara Simeoni*a, Simona Dinicola ${ }^{\mathrm{b}, \mathrm{c}}$, Alessandra Cucina ${ }^{\mathrm{c}}$, \\ Corrado Mascia ${ }^{\mathrm{d}}$, and Mariano Bizzarri ${ }^{\dagger \mathrm{e}}$ \\ ${ }^{a}$ Department of Mathematics, University of Nice Sophia Antipolis, Parc Valrose - 06108 \\ Nice Cedex 02, France \\ ${ }^{\mathrm{b}}$ Department of Clinical and Molecular Medicine, Sapienza University of Rome, Viale \\ Regina Elena 336 - 00161 Rome, Italy \\ ${ }^{\mathrm{c}}$ Department of Surgery "Pietro Valdoni", Sapienza University of Rome, via A. Scarpa \\ $14-00161$ Rome, Italy \\ ${ }^{\mathrm{d}}$ Department of Mathematics, Sapienza University of Rome, piazzale A. Moro $2-00185$ \\ Rome, Italy \\ ${ }^{\mathrm{e}}$ Department of Experimental Medicine, Sapienza University of Rome, via A. Scarpa 16 \\ - 00161 Rome, Italy
}

\begin{abstract}
In this report, we aim at presenting a viable strategy for the study of Epithelial-Mesenchymal Transition (EMT) and its opposite Mesenchymal-Epithelial Transition (MET) by means of a Systems Biology approach combined with a suitable Mathematical Modeling analysis. Precisely, it is shown how the presence of a metastable state, that is identified at a mesoscopic level of description, is crucial for making possible the appearance of a phase transition mechanism in the framework of fast-slow dynamics for Ordinary Differential Equations (ODEs).
\end{abstract}

Keywords: epithelial-mesenchymal transition, metastable states, systems biology, mesoscopic description, mathematical modeling, multiscale differential equations, slow-fast dynamics, stability analysis.

${ }^{*}$ corresponding author: simeoni@unice.fr

${ }^{\dagger}$ corresponding author: mariano.bizzarri@uniroma1.it 


\section{Introduction}

\subsection{Complex systems and phase transition}

Cell transition from a phenotype into another constitutes a critical event during development, differentiation and eventually the onset of degenerative diseases, like cancer. Phenotypic differentiation involves several changes at molecular, physiological and morphological level. Yet, rather than a progressive process, such transformation behaves like a first order phase transition, also involving the overall system in a coherent and global (phase) change.

A phase of a thermodynamic system and the states of matter typically have uniform physical properties. During a phase transition, certain properties of the given medium change, as a result of the variation of some external conditions (for example, temperature, pressure, or others). In Physics, first order phase transitions are characterized by a discontinuity in one or more state variables, and those we are particularly interested in also imply a change in entropy values [1]. By analogy, in biological systems, among the most reliable potential functions which describe such transitions, the Gibbs free energy plays a key role since its variations in response to the control parameters are usually mirrored by changes of the entropy.

Despite a number of factors have been demonstrated to participate into cell transitions - including stochastic genetic expression, physical and chemical forces - the cell differentiating process is still poorly understood.

The dynamics of a complex living system can be described at different levels of organization. The current mainstream posits that the lower level, that is the molecular one, exerts a privileged and even unique causative role in shaping how and why the basic units of life, cells and tissues, behave and develop [2]. The prevailing approach postulates that cell fate specification occurs as a deterministic process. In response to intrinsic and/or extrinsic chemical signals, a coordinated change in gene expression patterns drives the cell population into a specific differentiating pathway. This deterministic model has been widely criticized given that gene expression patterns are physiologically stochastic, and fluctuations increase even dramatically when the system (i.e., the cell population) is facing a critical transition from one stable differentiated state into another [3].

To reconcile the wide variability occurring at the microscale (i.e., molecular level) with the deterministic achievement of stable differentiated phe- 
notypes, the concept of epithelial plasticity has been introduced into the explanatory scheme [4]. This definition strives to capture two remarkable properties of living systems, namely resilience (robustness) to perturbations and extreme sensitivity to even small fluctuations of the environmental conditions.

A recurrent metaphor for the complex developmental path of cell systems across different phenotypic states is given by the Waddington landscape. In this model, cell phenotypes are depicted as stable attractors, also named as "valleys", while metastable or unstable states represent unstable attractors and are named as "hills" [5]. In view of the Mathematical Modeling of biological phase transitions we attempt at formalizing, a comment is in order about the semantic misunderstanding concerning the definition of metastable states. Actually, the geometrical characterization of such critical points is better illustrated by the denomination "saddle", and we shall employ the classical stability theory of dynamical systems [6] for the analytical study of the mathematical equations aiming at reproducing the biological experiments.

Stable states are usually identified by specific gene expression patterns and gene regulatory networks (GRNs) architecture. Indeed, the phasespace is reconstructed by computing GRNs from data provided by highthroughput experiments. However, because GRNs are typically intricate and contain highly nested feedback and feedforward loops that give rise to complex dynamics, it is difficult to elucidate cell behavior from these regulatory circuitries. Moreover, regulation of gene expression is currently no longer considered the causal factor driving cell differentiation [7]. A compelling body of evidence has shown that higher order factors efficiently constrain, and ultimately drive, processes occurring at lower scales [8, 9]. Such results have questioned the classical causative paradigm, deeply rooted into a reductionist, bottom-up approach. In addition, the non-linear interplay among factors belonging to different levels is highly sensitive to even smaller fluctuations in the initial conditions, or in other environmental parameters, thus providing the system with unexpected and unpredictable properties. This is why higher levels of matter aggregation display emerging properties that cannot be anticipated by fundamental laws or by analyzing single components, although the underlying enzimatic-genetic networks in a cell population also support the emergence of macroscopic structures.

Instead of focusing on the role of individual genes, proteins or pathways 
in biological phenomena, the aim of Systems Biology is to characterize the ways in which essential molecular parts interact with each others to determine the collective dynamics of the system as a whole.

Furthermore, regulation of the cell journey across the Waddington landscape may shed light to the emergence of complexity, and even into biological evolution. Indeed, it seems that complex forms of "organized" behavior in living matter emerge from the competition between different forms of order, rather than between species [10]. Therefore, as longer as conceptual categories such as order and complexity are involved in these processes, parameters like entropy and dissipative structures should be properly considered in any model of cell phenotypic commitment (refer to Section 3.3).

Thereby, to grasp physical emergent processes - namely, those occurring during phenotypic transitions, where the biological system is involved and changes coherently as a whole - we must look at the mesoscopic level/scale. By analogy with Physics, this is strongly affected by fluctuations around the average and subject to a probabilistic behavior. Indeed, it is mostly from such macroscopic changes that diseases, and especially cancer, are diagnosed.

\subsection{The mesoscopic framework}

The mesoscopic scale is the realm comprised between the nanometer and the micrometer, where "wonderful things start to occur that severely challenge our understanding" [11]. That is to say, at the mesoscopic level non-linear effects, as well as non-equilibrium processes, are more likely to be appreciated and "captured" [12]. Within that framework, both chemo-physical forces and boundary constraints can be deemed acting as causative factors, even if this property - the causal role - should be ascribed mostly to the very specific non-linear dynamics to which the different system components are subjected.

In Biology, the mesoscopic level usually entails both cells and tissues, and scientific investigation requires capturing pivotal features of these constituents. That approach also implies integrating different levels by focusing on parameters that display self-similarities at different scales (fractal dimension represents a paradigmatic case in point [13]). Through such a strategy, one would likely establish strict correlations between the local processes and the global structure of the living beings, by connecting every level with each other. It is worth noting that the topology (i.e., the geometrical threedimensional distribution) of the interacting components plays a critical role 
in shaping biological processes. Therefore, quantitative morphological analysis of both cells and tissues architecture has recently regained much interest, given that "the organization becomes cause in the matter" [14].

Furthermore, the mesoscopic framework shall provide an acceptable solution to the tyranny of scales problem, still a challenge to reductive explanations in both Physics and Biology [15]. The problem refers to the scaledependency of physical and biological behaviors, that often forces researchers to combine different models relying on different scale-specific mathematical strategies and boundary conditions. On the other hand, the mesoscopic approach outlines how coordinated (i.e., ordered) macroscale features and properties - including fractal morphology, cell population connectivity and motility, cytoskeleton rearrangement - arise from the collective behavior of microscale variables.

Those issues can be efficiently addressed by adopting a formalism (conceptual premises and framework) borrowed from the phase-space theory [16]. Indeed, the phenotypic differentiation is strongly reminiscent of phase transitions we observe in physical and chemical systems, and it is in fact formally equivalent when the non-linear dynamics features are properly taken into account [17]. From a mathematical point of view, the nonlinearity is mandatory to support the existence of multiple stationary states with various types of stability properties [6].

By analogy with phase transitions observed in inanimate matter, specific qualities of the biological system should be viewed as order parameters, and then their modifications are appreciated under the variation of a number of control parameters. As happens in Physics, also in Biology control parameters induce coherent changes in the system by involving it as a whole, that is to say by affecting "pleiotropically" a number of hypothetical targets (molecules and pathways, as well as cellular structures).

The transition from a state of order to a new one appears at the point of instability (bifurcation point), where the increased fluctuation in some of the order parameters leads to a transformation of the cell system, that displays long-range correlations and is self-similar at all scales of physical observation [18]. Order parameters, like the physical observables, thus enable in capturing the non-linear dynamics of the system. Moreover, a model based on those parameters shall overcome shortcomings represented by bottom-up modeling, on which reductionist approach usually relies. We strive to identify control parameters that drive the system to instability when approaching 
their critical values, and the resultant changes in the order parameters that correspond to the major physical modifications in the system under study.

The relevance of control parameters, usually belonging to description levels higher than the molecular one, has recently been vindicated by studies showing that cancer can be "reversed" through physical manipulation of the microenvironment [19]. For instance, it has been demonstrated that cell fate commitment in microgravity is largely dependent on the removal of physical (i.e., gravity) constraints [20]. Overall, such data strongly indicate that the stochastic non-linear dynamics governing processes at the molecular level can be efficiently and deterministically "constrained" and "ordered" by higher biophysical cues. The classical principle of causality is herewith addressed by taking into consideration those higher factors driving the system dynamics, hence recognized as control parameters, including external chemical stimuli, physical forces, environmental constraints and so forth.

Therefore, our central hypothesis is that the phenotypic transition may be described as a dynamical phase transition by considering only few system parameters and according to a multiscale approach. That model would allow capturing the critical points of the whole process to which further focused investigations are likely to unveil pivotal targets, eventually useful for therapeutically efficient intervention. The ultimate goal is to obtain a physico-chemical description of cell transition that could be translated into carcinogenesis studies, as cancer can be considered a "developmental process gone awry" [21].

\subsection{Epithelial-Mesenchymal Transition as metastable state}

Cells undergoing a phenotypic switch need preliminarily to enter into a metastable state, thus "destabilizing" their previous stable differentiated state. This destabilization is consistent with a first order critical transition, since suddenly opening access to new stable states - evoking a tipping point in the terminology of catastrophe theory [22, 23]. In correspondence to these points, the system experiences a wide fluctuation of many inherent parameters, including gene expression patterns [24].

A paradigmatic case in point is represented by the Epithelial-Mesenchymal Transition (EMT). Epithelial cells normally interact through specialized structures - mainly relying on E-cadherin-based "bridges" - as well as with basement membrane via their basal surface, thus being distributed within 
the surrounding space in a characteristic (fractal) manner. EMT is the biological process allowing such polarized cells to undergo multiple biochemical and/or structural changes that enable them to assume a mesenchymal cell phenotype, which includes enhanced migratory capacity, invasiveness, elevated resistance to apoptosis, and greatly increased production of ExtraCellular Matrix (ECM) components [25].

This transition occurs in a sufficiently dense population of cells (refer to Section 2.2 and involves the replacement of one group of cells - which originally adhere to each other forming a differentiated tissue - by another group of cells characterized by a highly heterogeneous and more motile aggregate. As such, EMT is a system process given that it is usually referred to a cell population sample, and can be assessed only at this level. Therefore, from a conceptual point of view, a Systems Biology approach is required to properly investigate EMT dynamics.

The transition from epithelial- to mesenchymal-cell characteristics encompasses a wide spectrum of inter- and intra-cellular changes, also involving the relationship among cells and with their microenvironment, thus representing a true modification of the whole system. It is remarkable that such transformation is reversible under specific environmental constraints, and it should be considered like a phase transition compatible with a mathematical formalization exhibiting a hysteresis loop (see Fig. 1 (a)).

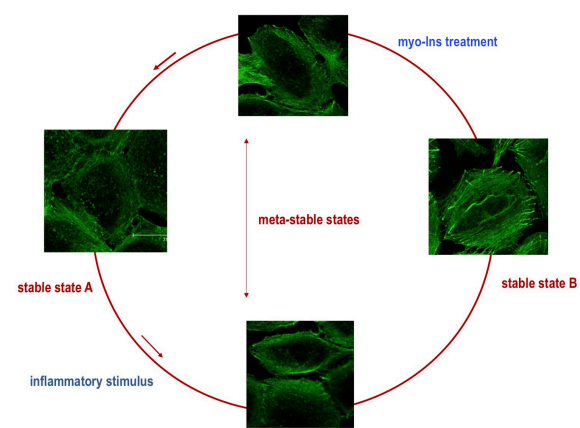

(a) Inflammatory stimulus and myo-Ins treatment effects on the EMT-MET process.

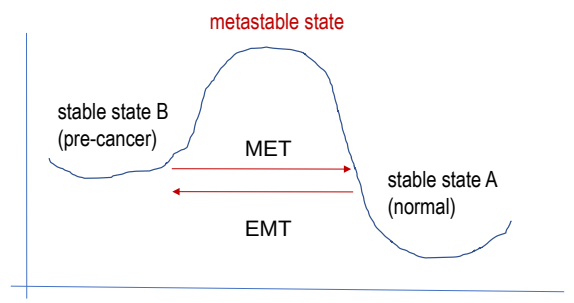

(b) An intermediate metastable state is necessary to accomplish a phase transition.

Figure 1: the EMT-MET schema.

Indeed, the reverse process, known as Mesenchymal-Epithelial Transition (MET), has also been reported [26], and promising studies on the "beneficial" effects of some external stimuli for inducing MET are in progress 
(see Fig. 21). Additionally, the recent discovery that MET is required for transforming somatic cells into pluripotent stem cells suggests that the intersection between EMT and MET is a fundamental crossroad for cell fate decisions [27].

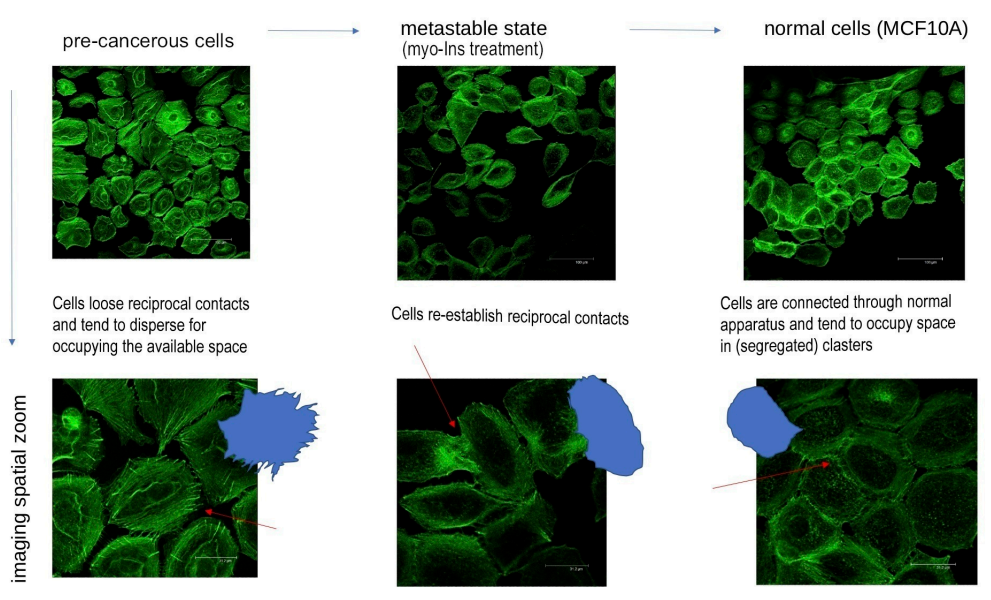

Figure 2: phenotypic reversal through myo-Ins-induced MET; schematic cell shape profiles are depicted as extracted from images, highlighting changes occurring during phenotypic transition.

Although such processes involve an overwhelming number of molecular factors and cellular structures [25], at the mesoscopic level a discrete number of parameters suffices for depicting the transition. Those parameters, mostly relying on (quantitative) changes entailing cell morphology and its dynamical relationships with the neighborhood, can be suitably considered as order parameters.

In this report, we aim at illustrating a methodological pathway for the phenomenon of phase-space transitions during cell fate specification, when a system passes from a stable state to another through a metastable bridge, having in mind the paradigmatic case of the EMT and MET. In that context, Mathematical Modeling provides an inherent texture for reality with the specific target of non-linear dynamics of diffuse information systems [28]. Also it is required to formalize external fields and boundary conditions which are determinant for the system dynamics, and to appreciate subtler system variations to predict more sophisticated behaviors (symmetry breaking, equilibria transition, ...). Mathematical Modeling may ultimately help recognizing critical factors and steps in promoting tumor reversal.

Two methodological directions are conceivable. Firstly, applied math- 
ematics for identifying and measuring the attractor manifolds for different equilibria by extrapolating information from experimental data. In that respect, the mathematical formulation of the biological problem helps in facilitating measurement quantifications rather than its qualification. Secondly, real-time multi-scale modeling to give evidence of phenomena with cumulative effects, for example models with memory terms and search for precursive factors to phase-space transitions. That approach can be performed at all description levels, from cells to organs passing through tissues, in order to induce medical actions starting from the theoretical analysis of precursive factors before the system moves too far from the healthy (stable) equilibrium (see Fig. 1(b)). It is worthwhile stressing that a stable dynamics should not be confused with a system in a stationary stable phase (namely, when nothing significant happens). Indeed, the former may anyway undergo a wide range of fluctuations without losing its stability. This means that a stable dynamics is characterized by resilience (robustness) with respect to external perturbations, given that it is located in the manifold of a stable attractor. On the contrary, a stationary stable system lies in a phase where no apparent dynamical changes occur.

Mathematical Modeling is asked to develop criteria to guide the interpretation of the observations in making "causes" and "effects" to raise from experiments (see Fig. 3). One wishes to identify lower order changes that are precursory to phase transitions inside the biological systems. In fact, identifying the metastable state during a complex biological process is a challenging task, because the state of the system may show neither apparent changes nor clear phenomena before a critical transition. Therefore, recognizing specific steps by means of additional mathematical variables which vary gradually could help, not only in identifying markers of transformation for early diagnosis, but also in determining drug targets.

The interaction between Systems Biology and Mathematical Modeling would have no hope of generating a virtuous circle, if not for the emergence of a new actor on stage: the computer. The performance development of modern computers has permitted to test models even remotely approachable in the past, through suitable numerical implementations [29]. By means of numerical algorithms, mathematical models so complex that they are not amenable of any rigorous analysis can be handled. In the biological field, we come even to coin a third experimental type class, adding to the experiments in vivo and in vitro also those in silico, with specific reference to computer 


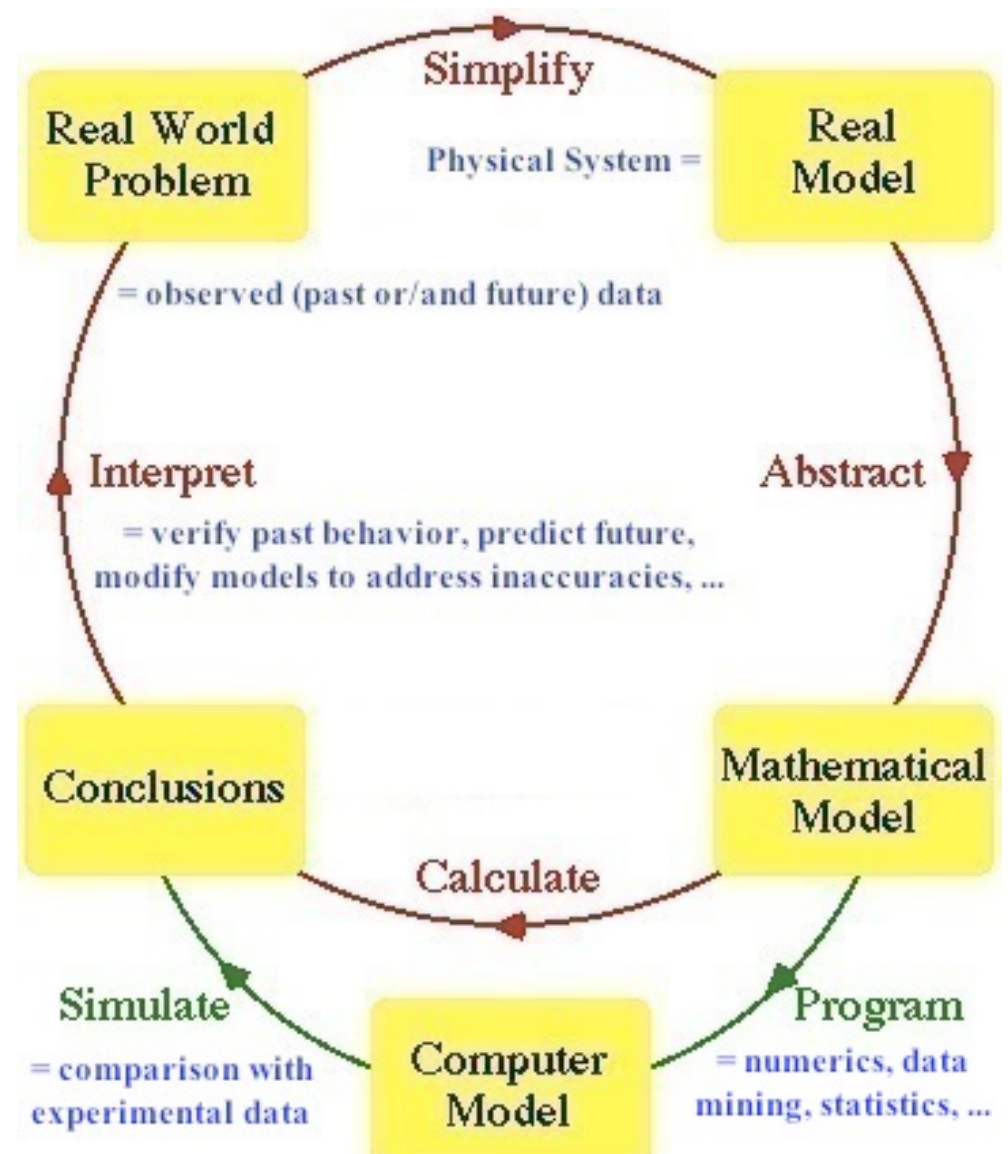

Figure 3: Mathematical Modeling pathway for adaptive design of biological experiments.

simulations (refer to Section 4).

\section{Material and methods}

\subsection{The experimental setting}

The experimental model is constituted by normal breast cells (MCF10A) that are exposed to micromolar concentrations of Transforming growth factor$\beta$ (Tgf- $\beta$ ), a well-known pro-inflammatory molecular effector [30]. As a result, MCF10A cells undergo a clear EMT within about 5 days - although preliminary effects can be appreciated already after 24-48 hours - by modifying their shape, the cytoskeleton architecture, the degree of inter-cellular relationships (with a significant reduction in E-cadherin based junctions), 
as well as their motility striving to occupy any available space (see Fig. 2). The Tgf- $\beta$-induced EMT should be considered a precursive step towards full transformation into fibrosis or an even worse (cancer) phenotype [31].

On the other hand, EMT is still a reversible process, which also exhibits an "intermediate" metastable state, that can be switched backward by appropriate changes in the control parameters. Indeed, by adding myo-Inositol (myo-Ins) treatments, the Tgf- $\beta$-induced EMT is almost reversed into a MET within 24-48 hours (see Fig. 1 (a)).

In what concerns the technical aspects of cell culture and reagents, the MCF10A breast cells line was purchased from the American Type Tissue Culture Collection (ATCC) and then cultured in a DMEM/F12 medium supplemented with $5 \%$ horse serum, $10 \mu \mathrm{g} / \mathrm{mL}$ insulin, $0.5 \mu \mathrm{g} / \mathrm{mL}$ hydrocortisone, $20 \mathrm{ng} / \mathrm{mL}$ EGF and $100 \mathrm{ng} / \mathrm{mL}$ cholera toxin. The cells were accompanied by $100 \mathrm{IU} / \mathrm{mL}$ penicillin and $100 \mu \mathrm{g} / \mathrm{mL}$ streptomycin, and kept in $5 \% \mathrm{CO} 2$ and humidified atmosphere at $37^{\circ} \mathrm{C}$. Recombinant human Tgf- $\beta 1$ was purchased from PeproTech and myo-Inositol was obtained from Lo.Li.pharma. About 3000 cells/well were originally plated, in a complete medium, onto micro cover glasses. Once at sub-confluent concentration, the cells were treated with $1 \mu \mathrm{L} / \mathrm{mL}$ of Tgf- $\beta 1$. After about 5 days, during which EMT occurred, the cells where stimulated with $4 \mathrm{mM}$ of myo-Inositol for 24 hours. As regards immunofluorescence, cellular morphology and F-actin ultrastructure have been investigated by adding phalloidin (Alexa Fluor 488) staining after cellular fixation with $4 \%$ paraformaldehyde and membrane permeabilization with ethanol and acetone in 1:1 ratio, and then visualized through confocal microscopy.

\subsection{Control parameters}

According to our experimental setting, cell-phase transition is triggered by two molecular signaling factors, acting essentially in opposite ways: Transforming growth factor- $\beta$ is a well-known inducer of EMT, while myo-Inositol has recently been demonstrated to be capable of inducing MET, thus counteracting the EMT opposite transformation [32].

The myo-Ins, a cyclic carbohydrate with six hydroxyl groups, is among the oldest components of living beings, undergoing complex evolutionary modifications ultimately leading to the current multiplicity of functions for Ins-containing molecules in eukaryotes [33]. While myo-Ins has no effect on normal (stable) cells, it significantly inhibits EMT in cells exposed to pro- 
inflammatory stimulation, as such provided by Tgf- $\beta$. This finding clearly suggests that myo-Ins effects start becoming apparent only at the bifurcation point, where the system undertakes the phase transition through a metastable state, near to symmetry breaking points [34].

Therefore, according to the formalism of phase-space transitions, both Tgf- $\beta$ and myo-Ins can be managed as control parameters.

The cell density should also be considered an "environmental" constraint. Indeed, experiments performed at different densities typically exhibit significant differences in terms of their results [35]. Changes in the cell density may actually influence cell-to-cell adhesion (thus modifying the overall connectivity of the cell population), stiffness and tensegrity response of the cell cluster (by modulating the mechano-transduction of a number of biophysical cues), and ultimately the shape acquired by cells [36].

\subsection{Order parameters}

As we have previously discussed, order parameters are measurable physical observables that allow representing the biological phenomenon. At the mesoscopic scale, a careful examination of the Tgf- $\beta$-induced EMT makes possible to extract a few key order parameters, which characterize crucial aspects of the experiments, including:

- Downregulation of E-cadherin (with reduced density values along the membrane border). Indeed, E-cadherin downregulation is a hallmark of the EMT and it constitutes a pre-requisite for cells committed towards transformation 37]. E-cadherin parameter evaluation epitomizes how different levels of observation are interconnected each other: on one side, E-cadherin can be quantified as an inter-molecular parameter (concentrations measured by western-blot assay within the cells); on the other side, E-cadherin distribution in discrete regions inside the cell can be appreciated by confocal (quantitative) microscopy, thus permitting its understanding as structural element. The combination of both these methods allows assessing the functional meaning of even subtle E-cadherin fluctuations. In addition, the correlation of raw Ecadherin concentration data with its specific localization inside the cell (in the membrane or cytosol domain) could actually provide the link between the sought molecular and structural levels of observation.

E-cadherin also participates, altogether with a number of other fac- 
tors, in the formation of cellular adhesion structures. In particular, its downregulation is responsible for reduced number of cell-to-cell adhesion foci experimentally observed [38]. Reduction in structural interand intra-molecular characteristics is among the most relevant cues that inhibit the constitution of a tissue and promote cells scattering in the available space.

- Shape changes and fractal dimension. Modification of the cell form usually entails the loss of apical-basolateral cell polarity, ultimately leading to substrate detachment. Cells detached from the substrate, as well as from their neighboring, are free to acquire new configurations and skills, including motility (see Fig. 4). Cell shape can be quantitatively assessed by means of a fractal approach and, in particular, fractal dimension (FD) is a well-suited marker of cell malignancy and motility [39].

Moreover, the fractal dimension epitomizes the morphological complexity of the overall cell system, by referring to the minimal required information for its description [40] and, at the same time, it allows to directly tackling the problem of multiple hierarchical levels. However, although the fractal dimension usually provides an indirect measure for the system entropy values, as extracted from quantitative morphological analysis, in the present context this value should not be confused with the internal entropy of the cell population.

In general, quantitative assessment of even subtle morphological changes has been proven to be predictive of further cell fate differentiation. In this respect, high-throughput time-lapse microscopy is a powerful tool for studying cell differentiation and bright-field imaging has been used to track and reconstruct cellular genealogies, namely through fluorescence-based recognition of molecular lineage markers [41]. However, molecular lineage markers are only available for few specific cell types, that are often already differentiated, thus hindering the early identification of differentiating cells. On the other hand, a few attempts have already been made to extract and exploit the information embedded in confocal microscopy images for prospective detection of lineage commitment.

- Cytoskeleton rearrangement and stress fibers. The cytoskeleton (CSK), especially through F-actin remodeling, promotes both new shape con- 

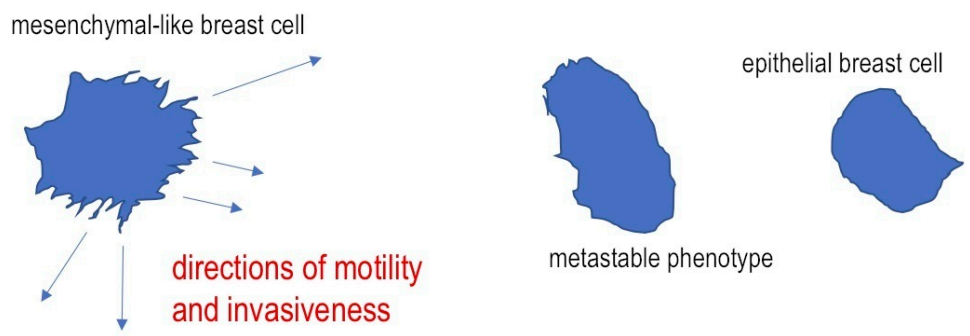

Figure 4: parallel changes in cell shape and phenotypic plasticity highlighting how the more migrating/invading cells display higher fractal dimension.

figurations and selective activation of a number of genetic and biochemical pathways. Overall, changes in CSK can be appreciated by means of an integrated parameter, named coherency, that extracts the relative strength of the edges of structures compared to their surroundings. Therefore, it should be considered like a measure of "the global alterations in the organization of the F-actin" [42].

Appearance of stress fibers is quantitatively assessed, as for other CSK components, through confocal microscopy. Stress fibers have been shown to play an important role in cellular contractility, migration and invasiveness, especially during EMT [43]. This process ultimately ends up in the emergence of filopodia and pseudopodia, indicating increase motility and invading capacity (see Fig. 5). These structures are mechanistically linked to CSK and to the cell membrane, allowing cells to perform many specialized functions (invasion of the ECM, motility, exploration of the surrounding space).

An important fact is that the aforementioned parameters are "independent" each other, and they cannot be replaced from one another. Yet, they are not exclusive given that various order parameters, tightly correlated with the same features we are looking at, could have been also taken into account (refer to Section 4) and shall further be incorporated for improving the Mathematical Modeling.

It is wortwhile stressing that, in our experimental setting, normal cells in culture are usually "confined" into clusters and they do not display significant spreading. Moreover, during the first 24-48 hours of culture, the mitotic and apoptotic rates do not change significantly, so that the cell density (cells per area) can reliably be considered as fixed. Together with the fact that density influences in a crucial way the experimental development, 


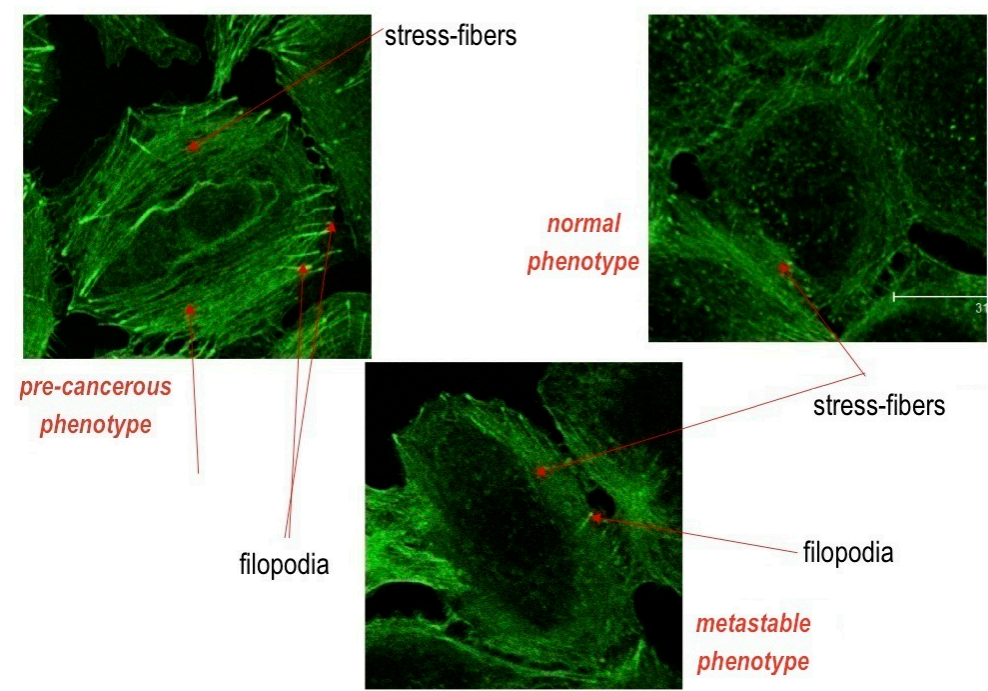

Figure 5: different CSK configurations supporting distinct cell phenotypes.

this justifies its role as control parameter.

\section{Modeling}

\subsection{The mathematical framework}

Next, we attempt at listing crucial features of the mathematical model to reproduce the biological problem described above.

- ODEs and time-discrete approximation. The experiments are essentially time-dependent, and changes of the distribution of cells in space and number (density) could be considered constant, at a first modeling stage. That assumption would be satisfied in agreement with the experimental conditions we set for our model, in particular low values of fetal bovine serum (FBS) added to the culture medium (refer to Section (4). Indeed, low FBS concentration implies that cells are only minimally stimulated, and thus display negligible growth rate and migratory capabilities.

Therefore, the mathematical models are constituted by systems of Ordinary Differential Equations (ODEs) with the eventual presence of stochastic terms [44]. In addition, time-discrete approximations could be developed, in order to perform numerical simulations for comparison with the experimental data (refer to Section 4). As a matter 
of fact, since the evaluations of the biological process are typically conducted at discrete time instants, one could also directly formalize time-discrete models (i.e., recurrence equations) from which appropriate ODEs are deduced by taking times-continuous limits [45].

- Space dependency. Nevertheless, space dependency is relevant: since our target is to "revert" potentially malignant cells earlier, before they acquire a migrative and invasive phenotype, the space rather plays the role of an external parameter in the sense that important properties of the cell population manifest a space dependency (density, lacunarity, critical malignant features, ...) although without transport terms and/or spatial gradients.

Moreover, the experimental setting presupposes initial conditions with cells uniformly distributed and synchronized over the culture support, but however slight differences in the cell cycle cannot be avoided, and thus space inhomogeneoities have to be taken into account.

- Slow-fast dynamics. The transition time for EMT and MET is typically very short with respect to the overall lifetime of the biological system. This translates into the fact that the corresponding mathematical model should exhibit a slow-fast decomposition [46].

More precisely, we require that the differential equations incorporate a small parameter $\tau \geq 0$ governing the time-scale, so that, for infinitely small values of such parameter, namely as $\tau \rightarrow 0^{+}$(the so-called singular perturbation limit), we recover the qualities of a first order phase transition. A major consequence of this approach is that the ODEs system still hold for strictly positive values of the time-scale parameter, hence providing a reliable description also for second order phase transitions with $\tau \gtrless 0$.

- Multi-scale approach. Phase transitions are described by means of a multi-scale model. Some observable parameters are actually averages of microscopic quantities and can be further mirrored by the behavior of lower order parameters. Within its general structure, our mathematical formalization do not restrain from taking into account genetic or other microscopic factors (GRNs, for example). Systems Biology considers external forces which are integrated to the various levels for having effects on the cells, then the feedbacks inside the system are essential ingredients for adequate models. Several math- 
ematical strategies allow to relate passages from different space-time levels and different scales can be effectively included: hydrodynamical limits from cells to tissues, integro-differential equations for memory terms and non-local issues, and asymptotic analysis, among others.

- Entropy and fractal analysis. In biological systems, fluctuations in the amount of entropy can be equated, at a first glance, to variations of the Gibbs free energy. In turn, changes in entropy values can be tracked by evaluating modifications in the fractal properties of the cell system 47, 48]. Various formulae for the fractal dimension of biological systems are in fact defined based on entropy functions [49]. It is worth recalling that entropy evaluation always depends on the scale of measurement, thus resulting in uncertainty, whilst the fractal dimension is independent of (discrete) measurement scales.

From a mathematical point of view, we aim at identifying a global (space- and time-dependent) function, the so-called Lyapunov functional, accounting for the overall "stress" of the dynamical process [6], and try to determine the points where this function experiences a symmetry breaking so that the system starts transiting towards metastable states (refer to Section 1.3). The variational analysis of auxiliary quantities different from the order parameters, which have eventually varied when the system leaves an equilibrium, would provide the precursive signature of a phase-space transition.

\subsection{Formal equations}

Let us consider the vector (i.e., collection) of physical variables $V=(E, F, C)$, where $E, F, C$ stand for system-averaged values of E-cadherin, fractal dimension and coherency, respectively. We assume that the dynamics of the cell system is justly characterized by the time evolution of these quantities. The choice of those order parameters for reproducing the biological experiments is not exclusive, and the same mathematical formalism could also be adopted for other observable quantities (refer to Section 4).

Then, the experimental setting is translated into a set of first order ODEs for the instantaneous time variation of the order parameters, which is denoted by

$$
\frac{d V}{d t}=\left(\frac{d E}{d t}, \frac{d F}{d t}, \frac{d C}{d t}\right)
$$


and should be interpreted as time-derivative in mathematical language.

For time $t$ varying between 0 and about 5 days (starting and ending of the biological experiment), the differential model reads

$$
\frac{d V}{d t}=\Phi(V ; S)
$$

for some (vector-valued) structural function $\Phi=\left(\Phi_{1}, \Phi_{2}, \Phi_{3}\right)$ describing the biological mechanism underlying the dynamical process, and with $S$ representing the external stimuli (i.e., control parameters), that include inflammatory factors, myo-Ins, cell density, physical constraints and other eventual terms. The equation (1) can be rewritten in scalar components as

$$
\left\{\begin{array}{l}
\frac{d E}{d t}=\Phi_{1}(E, F, C ; S) \\
\frac{d F}{d t}=\Phi_{2}(E, F, C ; S) \\
\frac{d C}{d t}=\Phi_{3}(E, F, C ; S)
\end{array}\right.
$$

and it must be complemented with appropriate initial conditions $E(0)=E_{0}$, $F(0)=F_{0}$ and $C(0)=C_{0}$ to be deduced from the experimental measures for $E_{0}, F_{0}$ and $C_{0}$. On the other hand, since $S$ embodies the control parameters, it should be considered as a known function which may be constant or rather time- and space-dependent (for example, if growth factors or treatments are administered at specific discrete temporal instants or/and in a spatial nonhomogeneous way to the population of cells).

Concerning the space dependency, we choose a two-dimensional reference domain $\Omega \subset \mathbb{R}^{2}$ corresponding, for example, to a Petri dish or any technical support where the cell culture is analyzed (see Fig. 6). In principle, similar statements hold in the physical three-dimensional space.

Due to the high number of cells involved in the biological trials, a tissue-like behavior emerges for the whole system, and thus the hypothesis of a spacecontinuous description is pertinent. Hence, system-averaged values of $E, F$ and $C$ can be defined in terms of the corresponding cell-related "densities" as the following spatial integrals

$$
\begin{aligned}
& E(t)=\frac{1}{|\Omega|} \int_{\Omega} e(t, \mathbf{x}) d \mathbf{x}, \\
& F(t)=\frac{1}{|\Omega|} \int_{\Omega} f(t, \mathbf{x}) d \mathbf{x}, \\
& C(t)=\frac{1}{|\Omega|} \int_{\Omega} c(t, \mathbf{x}) d \mathbf{x},
\end{aligned}
$$




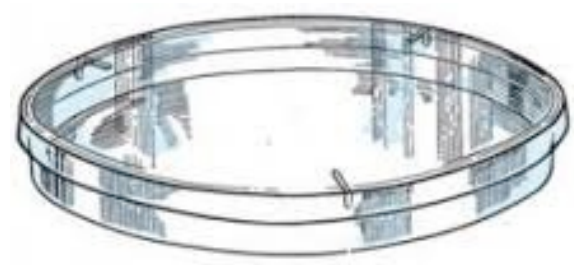

(a) A circular Petri dish.

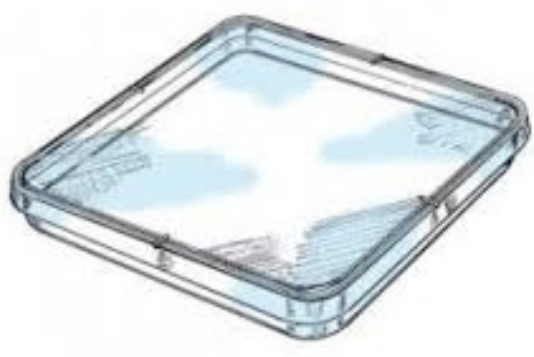

(b) A squared Petri dish.

Figure 6: two examples of cell culture plates.

with $|\Omega|$ denoting the area of the experimental domain. Here, for time $t \geq 0$ and position $\mathbf{x} \in \Omega$, the functions $e, f$ and $c$ describe the density of E-cadherin, fractal dimension and coherency, respectively, and they are introduced to take into account the microscopic features of the cell system. This constitutes a first instance of multi-scale approach since different levels of observation - specifically, from cells to tissues - are mathematically related. Indeed, a model similar to (2) can be formulated also at the microscopic scale, namely

$$
\left\{\begin{array}{l}
\frac{d e}{d t}=\varphi_{1}(e, f, c ; S) \\
\frac{d f}{d t}=\varphi_{2}(e, f, c ; S) \\
\frac{d c}{d t}=\varphi_{3}(e, f, c ; S)
\end{array}\right.
$$

so that the macroscopic equations (2) are recovered through space-averaged integrals (3) provided that the structural functions $\varphi_{1}, \varphi_{2}$ and $\varphi_{3}$ in (4) are properly designated. Although intrinsically coherent with a multi-scale framework, such procedure could be extremely intricated to be performed in practical cases, especially when the control parameters $S$ are spacedependent. Nevertheless, unlike the global/macroscopic order parameters $E, F$ and $C$ which are naturally defined for the whole system by extracting information from the corresponding local/microscopic densities $e, f$ and $c$ (refer to Section 2.3), the control parameters $S$ are more efficiently established directly at a higher order (i.e., mesoscopic) level, without the necessity of moving down to the microscopic scale. Obviously, that strategy does not exclude from considering the microscopic processes induced on the cells by the presence of those external stimuli - including genetic expression, physical and chemical molecular forces - by formulating explicit forms for $\varphi_{1}, \varphi_{2}$ 
and $\varphi_{3}$ in (4), but this is not mandatory for the success of our approach.

The minimal requirement for the vectorial model (1) or, equivalently, for its component-wise version (2) to represent an acceptable candidate for modeling EMT processes is that they displays three stationary solutions, two stable states and one unstable/metastable state (see Fig. 11). This forces the function $\Phi$ and its components $\Phi_{1}, \Phi_{2}$ and $\Phi_{3}$ to satisfy some essential structural conditions, in order to ensure that

$$
\Phi(V ; S)=0 \quad \Longleftrightarrow \quad V \in\{A, B, M\} \quad \text { for any } S
$$

or, equivalently,

$$
\left\{\begin{array}{l}
\Phi_{1}(E, F, C ; S)=0 \\
\Phi_{2}(E, F, C ; S)=0 \\
\Phi_{3}(E, F, C ; S)=0
\end{array} \quad \Longleftrightarrow \quad(E, F, C) \in\{A, B, M\} \quad \text { for any } S\right.
$$

for some (distinct) vectors $A=\left(E^{A}, F^{A}, C^{A}\right), B=\left(E^{B}, F^{B}, C^{B}\right)$ and $M=$ $\left(E^{M}, F^{M}, C^{M}\right)$ corresponding to biologically relevant equilibria.

Additional conditions guaranteeing stability for $A$ and $B$, and metastability for $M$, have also to be satisfied (refer to Section 3.3).

Since the equilibrium system (5)-(6) is multi-dimensional, the phase-space exhibits a non-trivial geometrical landscape (see Fig. 7), and transitions can occur with sudden change of values concerning only some variables (like for contact discontinuities in continuum physics [50]).

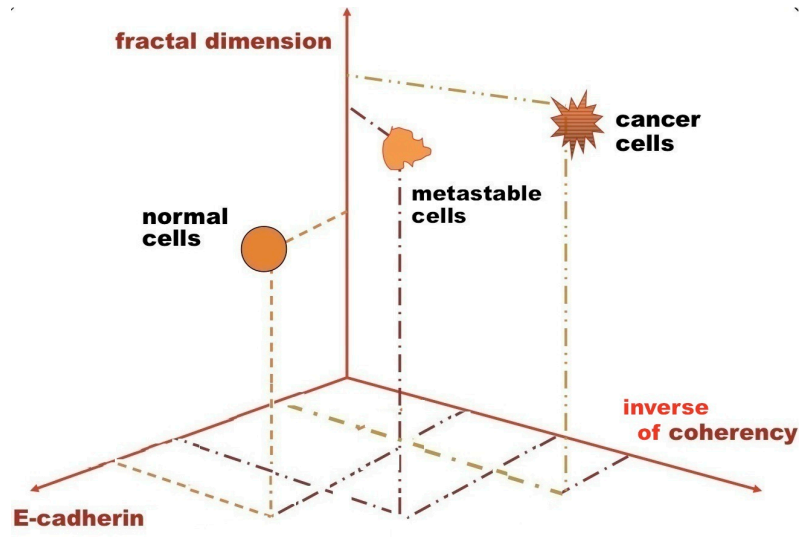

Figure 7: hypothetical three-dimensional space-phase diagram depicting the performance of order parameters. 


\subsection{A tutorial example}

We consider a simplified model consisting of a (nonlinear) system with two coupled first order ODEs for the variables $u$ and $w$, that is

$$
\tau \frac{d u}{d t}+u=w, \quad \frac{d w}{d t}+w=\lambda g(u),
$$

where the external constraints are given by constant parameters $\tau, \lambda>0$ and $g$ is a known structural function whose properties are detailed later on. In comparison with the general model (1)-(2), order and control parameters correspond to $V=(u, w)$ and $S=(\tau, \lambda)$, respectively, and

$$
\Phi_{1}(u, w ; \tau, \lambda)=\frac{1}{\tau}(w-u), \quad \Phi_{2}(u, w ; \tau, \lambda)=\lambda g(u)-w .
$$

We attempt at formulating a hypothetical interpretation of the dynamical process (7) in terms of biological observations, assuming that $u$ represents E-cadherin boundary values and $w$ stands for the coherency, which is connected with relative E-cadherin density values along the membrane border with respect to its overall concentration. Then, the specific expression for $\Phi_{1}$ encodes the fact that $u$ - describing the E-cadherin boundary distribution of the cell population - tends to conform to the behavior of $w$ - accounting for the system coherency - in a (typically fast) time-scale of order $\tau$. Similarly, the expression for $\Phi_{2}$ entails the convergence of $w$ towards $\lambda g(u)$ in a (slower) time-scale of order 1.

According to the abstract calculations in (5)-(6), that now translate into

$$
\left\{\begin{array}{l}
\Phi_{1}(u, w ; \tau, \lambda)=0 \\
\Phi_{2}(u, w ; \tau, \lambda)=0
\end{array}\right.
$$

for the specific functions (8), the stationary solutions to (7) are given by the points $(u, w)$ which are located at the intersection of the curves

$$
w=u \quad \text { and } \quad \lambda g(u)=w
$$

laying on the phase-plane (i.e., the two-dimensional projection of the phasespace). As a consequence, the set of equilibria for the dynamical system (7) is characterized, for any fixed $\lambda>0$, as the zeros of the function (see Fig. 8(b))

$$
h(u ; \lambda):=u-\lambda g(u)
$$

and the stability properties are deduced by analyzing its first order derivative (namely, the first order variation of $h$ with respect to $u$ ), that is

$$
\frac{d h}{d u}(u ; \lambda)=1-\lambda \frac{d g}{d u}(u) .
$$




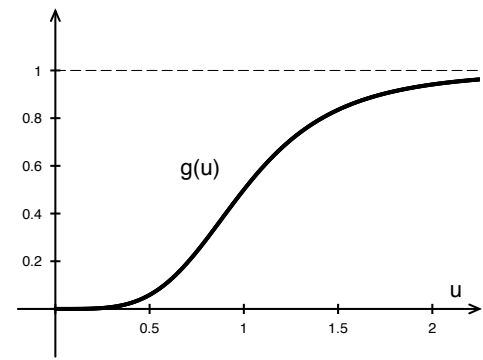

(a) A typical S-shaped function $g$ with $p=1$ and $\ell=1$.

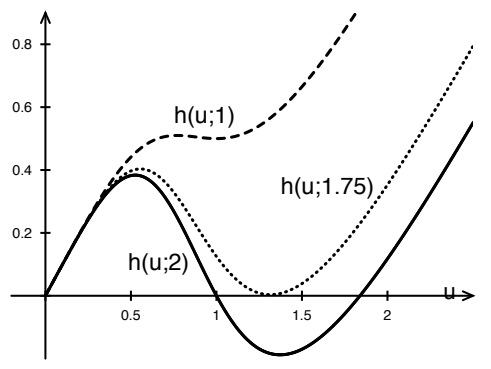

(b) The corresponding function $h$ for distinct values of $\lambda(\lambda=1$ dashed, $\lambda=\lambda_{c}=1.75$ dotted, $\lambda=2$ continuous).

Figure 8: the graphs of the structural functions $g$ and $h$.

Actually, for the particular case of system (7), this approach is equivalent to the standard spectral analysis (refer to Section 44). More precisely, if the derivative $\frac{d h}{d u}(u ; \lambda)$ is positive, the equilibrium is stable; otherwise, if it is negative, the equilibrium is unstable/metastable. Under the assumption that $g$ is non-decreasing (namely, $\frac{d g}{d u}(u) \geq 0$ for any $u$ ) and such that $g(0)=$ $\frac{d g}{d u}(0)=0$, the origin of the phase-plane $(u, w)=(0,0)$ is a solution to (9) and, moreover, it is a stable equilibrium because $\frac{d h}{d u}(0 ; \lambda)=1$ for any $\lambda>0$ from equation 10 .

In terms of biological experiments, the stationary state $(0,0)$ satisfying the above conditions could be associated with the original (unperturbed) phase of the system (normal cells). Besides, due to the nonlinearity of the function $g$, the mathematical description (7) also incorporates the existence of other biological equilibria - different from $(0,0)$ - corresponding to further phases of the cell system during EMT or/and MET (refer to Section 1.3). Indeed, according to (9), any eventual subsequent intersection between the curve $w=\lambda g(u)$ and the straight line $w=u$ gives raise to additional equilibria, alternating stable and unstable/metastable states in the case of simple zeros of $h$ (which occur under the generic assumption that $h(u ; \lambda)=0$ implies $\frac{d h}{d u}(u ; \lambda) \neq 0$, that is the so-called transversality condition).

Then, we conjecture that $g$ behaves like an S-shaped function, meaning that $g$ is convex in the interval $(0, p)$ and concave in its complement $(p,+\infty)$ for some $p>0$, and its values are bounded from above, so that $g(+\infty)=\ell$ for some threshold $\ell>0$ (see Fig. 8(a)). Therefore, two distinct ranges 
of values for the control parameter $\lambda$ can be considered, leading to quite different emerging scenarios (see Fig. 8(b)) classified as follows:

- small $\lambda$, corresponding to a unique equilibrium, given by $(u, w)=(0,0)$;

- large $\lambda$, that is consistent with the presence of three intersection points (i.e., equilibria).

These two regimes are separated by a (non-generic) critical value $\lambda=\lambda_{c}>0$ which produces only two distinct equilibria.

In view of the previous analysis, one infers that model (7) is, at the same time, minimal and reliable. Indeed, small values of $\lambda$ (i.e., $\lambda<\lambda_{c}$, see Fig. 9(a)) illustrate a biological situation where the external physical constraints - for example, inflammatory factors or myo-Ins treatments - are too weak for determining any phase transition, hence the system remains in its original (healthy or pre-cancerous) configuration. On the other hand, for large $\lambda$ (i.e., $\lambda>\lambda_{c}$, see Fig. $\left.9(b)\right)$ the mathematical system supports phase transitions alternating stable and metastable states, and the possibility of simulating EMT or/and MET with the typical "destabilization mechanism" introducing a metastable state (refer to Section 1.3). The importance of identifying, and also quantifying, the critical threshold $\lambda_{c}$ appears, in particular, when medical actions have finally to be undertaken, because the control parameters can be adjusted to predictively regulate the system response.

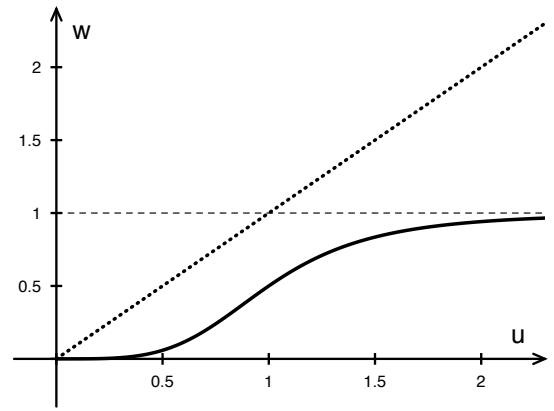

(a) Case $\lambda=1<\lambda_{c}$ with $p=1, \ell=1$.

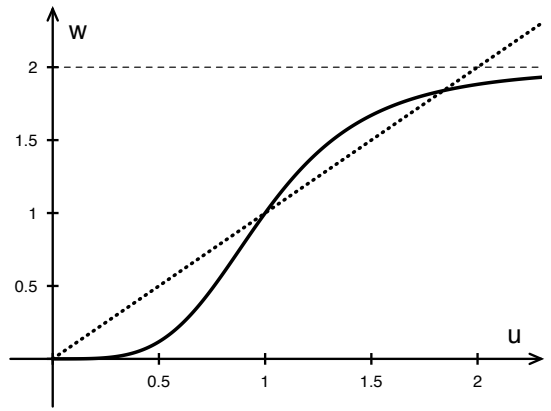

(b) Case $\lambda=2>\lambda_{c}$ with $p=1, \ell=1$.

Figure 9: relative positions of the straight line $w=u$ (dotted) and the curve $w=\lambda g(u)$ (continuous) corresponding to different values of $\lambda$.

It is worthwhile noticing that multiple equilibria could be generated by assuming other forms for the function $g$, thus allowing to establish effective 
mathematical models for reproducing a wide variety of biological dynamics.

The fast-dynamics of (7) is described by a new time variable $s$ related to the old one $t$ by the relationship $s=t / \tau$, which corresponds to the following differential equality for the instantaneous time variation:

$$
\frac{d}{d s}=\tau \frac{d}{d t} .
$$

This procedure consists in rescaling the time variable in order to zoom in on the system process during the first temporal period. Passing from $t$ to $s$, the system of ODEs becomes

$$
\frac{d u}{d s}+u=w, \quad \frac{d w}{d s}+\tau w=\tau \lambda g(u)
$$

and its limit as $\tau \rightarrow 0^{+}$is formally given by

$$
\frac{d u}{d s}+u=w, \quad \frac{d w}{d s} \approx 0 .
$$

The second equation in (11) does trivially express the fact that $w$ is, at first glance, indipendent of $s$ (i.e., constant). Therefore, the corresponding approximated solutions to (11) are

$$
\frac{d u}{d s}+u \approx w_{0}, \quad w \approx w_{0},
$$

and, by applying classical results on explicit solutions to linear ODEs [51], one ultimately obtains

$$
u(s) \approx w_{0}+\left(u_{0}-w_{0}\right) e^{-s}, \quad w(s) \approx w_{0},
$$

for some initial conditions $u(0)=u_{0}$ and $w(0)=w_{0}$. Finally, in a fast timescale ( $s \rightarrow+\infty$ or, equivalently, $\tau \rightarrow 0^{+}$) the solution gets closer and closer to the straight line $w=u$. The "fate" of the system is not yet decided, but it appears to be dictated only by the variable $u$, whose dynamics is determined at a slower time-scale. Coming back to equations (7) and putting formally $\tau=0$, we deduce that the slow dynamics is described by the reduced system

$$
u=w, \quad \frac{d w}{d t}+w=\lambda g(u)
$$

which corresponds to the scalar equation

$$
\frac{d u}{d t}+u-\lambda g(u)=0
$$


The characterization of the equilibria for 12 - 13 and their stability analysis is precisely what has been performed above, recalling that $h(u ; \lambda)=$ $u-\lambda g(u)$.

A remarkable fact is that system (7) possesses an alternative representation consisting of a single second-order differential equation, which can be obtained by differentiating the first equation of (7) with respect to time $t$ and taking advantage of the second equation, so that

$$
\tau \frac{d^{2} u}{d t^{2}}+\frac{d u}{d t}=\frac{d w}{d t}=\lambda g(u)-w
$$

and then first equation is used again to obtain

$$
\tau \frac{d^{2} u}{d t^{2}}+(1+\tau) \frac{d u}{d t}+u-\lambda g(u)=0,
$$

which is known as the one-field equation. Multiplying equation (14) by the first order derivative $\frac{d u}{d t}$ and applying the chain rule give

$$
\underbrace{\tau \frac{d^{2} u}{d t^{2}} \frac{d u}{d t}}_{\frac{d}{d t}\left\{\frac{\tau}{2}\left(\frac{d u}{d t}\right)^{2}\right\}}+(1+\tau)\left(\frac{d u}{d t}\right)^{2}+\underbrace{\{u-\lambda g(u)\} \frac{d u}{d t}}_{\frac{d}{d t}\left\{\frac{1}{2} u^{2}-\lambda G(u)\right\}}=0,
$$

where $G$ denotes a primitive of $g$ (i.e., a function such that $\frac{d G}{d u}(u)=g(u)$ for any $u$ ). Equation (15) shows the dissipative structure of the dynamics: indeed, this can be rewritten as

$$
\frac{d}{d t}\left\{\frac{\tau}{2}\left(\frac{d u}{d t}\right)^{2}+\frac{1}{2} u^{2}-\lambda G(u)\right\}=-(1+\tau)\left(\frac{d u}{d t}\right)^{2},
$$

where the quantity under the time-derivative has a negative variation, and thus decreases in time. Therefore, the term

$$
\frac{\tau}{2}\left(\frac{d u}{d t}\right)^{2}+\frac{1}{2} u^{2}-\lambda G(u)
$$

is a Lyapunov functional for the equation (14), and thus it is designated for being an intrinsic entropy for the dynamical process (refer to Section 3.1).

\section{Notes}

- Order parameters. In principle, we could have chosen different molecular parameters in the place of E-cadherin. However, besides the specific 
relevance of E-cadherin during EMT, most of these parameters cannot be considered as being independent with respect to the E-cadherin. For example, the N-cadherin - a paradigmatic marker of mesenchymal transformation - increases or decreases exactly in opposite way to Ecadherin. Similarly, the Focal Adhesion Kinases (FAK) or $\beta$-catenin membrane density, are, in some way, related to the E-cadherin. By including these parameters, no eloquent "information" would be further added to the model.

- Cell culture protocols. Currently, a number of artifacts frequently biases cell culture models. For instance, cells are typically stressed by high concentrations of growth factors, which are added to the culture medium to promote sustained proliferation. As a matter of fact, this "accelerated" growth regimen could likely overcome regulatory loops by introducing into the system an additional, unwarranted and usually overlooked, control parameter (i.e., external stimulus). Therefore, we conditioned MCF10A cells growing in a medium supplemented with low FBS levels (1\%) to avoid undue metabolic and proliferative consequences. Moreover, low-FBS regimen - without impairing cell viability - kept cell density in a quasi-stationary state for at least 24-48 hours, with minimal change in cell population count (refer to Section 2.3.

- Numerical algorithms. Especially to reproduce the outcome of in vitro experiments, it is pertinent to have recourse to scalar-valued equations settled on a two-dimensional domain $\Omega \subset \mathbb{R}^{2}$ with regular boundary (see Fig. 6), although the approach developed in this report straightforwardly extends to systems in the three-dimensional space. In order to perform numerical simulations for comparison with the experimental data, time-discrete approximations have to be developed, and spatial finite differences on staggered grids can be applied for dealing with the space dependency [52, 53]. The Runge-Kutta method is particularly suitable for the numerical simulation of time-evolution differential equations [45]. In general, time-implicit schemes are quite computationally inefficient for complex problems and, indeed, highorder Runge-Kutta time-integration solvers are important tools for improving the resolution of explicit simulations. On the other hand, the importance of designing spatially compact difference operators is 
motivated by the requirement of an optimal implementation in parallel computers [54, 55]. in fact, since the nearest-neighbor communication standard is extremely fast with the need of small amounts of local storage in the sub-processors (as only few values are involved to update the numerical solution at each grid point), even very large models becomes feasible, thanks to the massive number of threads especially in GPU-based computing devices [56]. For the sake of completeness, we mention that a modern $\mathrm{C}++$ library for numerically solving ODEs is available at www. odeint.com - which is compatible with running on CUDA GPUs programming architecture through the Thrust interface available at http://thrust.github.io

- Linearized operator and spectral analysis. The dynamical system (7) is nonlinear because of the presence of the nonlinear term $g(u)$ inside the second equation, which is responsible for the existence of multiple non-trivial equilibria (refer to Section 3.2). The linearization at an equilibrium point $(u, w)$ gives a system for the first order perturbation $(\xi, \eta)$ that is

$$
\frac{d \xi}{d t}=\frac{1}{\tau}(-\xi+\eta), \quad \frac{d \eta}{d t}=\lambda \frac{d g}{d u}(u) \xi-\eta,
$$

or, in vectorial form,

$$
\frac{d}{d t}\left(\begin{array}{l}
\xi \\
\eta
\end{array}\right)=\mathbb{A}\left(\begin{array}{l}
\xi \\
\eta
\end{array}\right)
$$

where the matrix

$$
\mathbb{A}=\left(\begin{array}{cc}
-\frac{1}{\tau} & \frac{1}{\tau} \\
\lambda \frac{d g}{d u}(u) & -1
\end{array}\right)
$$

is known as the jacobian matrix. Spectral analysis is based on the computation of the eigenvalues (and, specifically, on their sign) of $\mathbb{A}$, which are the roots of the characteristic polynomial given by

$$
\begin{aligned}
p(\mu) & :=\operatorname{det}(\mathbb{A}-\mu I)=\left(-\frac{1}{\tau}-\mu\right)(-1-\mu)-\frac{\lambda}{\tau} \frac{d g}{d u}(u) \\
& =\mu^{2}+\left(1+\frac{1}{\tau}\right) \mu+\frac{1}{\tau}\left(1-\lambda \frac{d g}{d u}(u)\right) .
\end{aligned}
$$

Denoting by $\mu_{1}$ and $\mu_{2}$ the zeros of the above polynomial, the following representation holds

$$
p(\mu)=\left(\mu-\mu_{1}\right)\left(\mu-\mu_{2}\right)=\mu^{2}-\left(\mu_{1}+\mu_{2}\right) \mu+\mu_{1} \mu_{2},
$$


and therefore, comparing with (17), we deduce that

$$
\mu_{1}+\mu_{2}=-\left(1+\frac{1}{\tau}\right), \quad \mu_{1} \mu_{2}=\frac{1}{\tau}\left(1-\lambda \frac{d g}{d u}(u)\right) .
$$

Recalling that $\frac{d h}{d u}(u ; \lambda)=1-\lambda \frac{d g}{d u}(u)$ from $(10)$, if $\frac{d h}{d u}(u ; \lambda)$ is positive, the product $\mu_{1} \mu_{2}$ of the two roots is positive - indicating that they have the same sign - and their sum $\mu_{1}+\mu_{2}$ is negative - indicating that they are both negative - so that the equilibrium state $(u, w)$ is stable. Complementarily, if $\frac{d h}{d u}(u ; \lambda)$ is negative, one root is positive and the other is negative, consistently with the appearance of a saddle point, or, in other words, a metastable equilibrium. The above fact is a special form of the more general Routh-Hurwitz criterion [57].

\section{Conclusions and perspectives}

Reproducibility of the results presented in this report has been assessed by means of triplicate, independent experiments. Indeed, Tgf- $\beta$ induced EMT is always obtained after 5 days of treatment, involving up to $90 \%$ of cells as recorded by molecular and morphological analyses. Similarly, myo-Ins induced MET occurs after 24 hours by involving up to $85 \%$ of transformed cells. In addition, the analysis of the mathematical models may suggest new features for the experimental setting, also by means of numerical simulations for enlarged models obtained by adding terms, factors and mechanisms which are further developments of the biological experiments. One could also postulate auxiliary order parameters, for example mathematical derivatives of the principal functions, to earlier predict phase transitions with the ultimate target of designing external controls to prevent such transitions.

It is extremely important that mathematical models capture the emergence of dynamics at higher levels, since the behaviour of the system is not merely the result of the collective evolution of its isolate components, but it proceeds from the effect of (global) constraints. This emphasizes the intrinsic limits of studying biological phenomena on the basis of purely microscopic experiments (indeterminateness of measurements, instantaneous time,...) and, therefore, a multi-scale model (with some parameters derived from the microscopic analysis) is better suited from a methodological point of view. In that context, the so-called emerging properties are interpreted 
as systemic averages of microscopic behaviours (for example, the effects of the inositol on the density of breast tissues has been measured before understanding its microscopical chemical reactions).

\section{Acknowledgments}

The results presented in this report have been obtained in the framework of the Working Group on Phase Transitions in Biology through Mathematical Modeling, settled at the Systems Biology Group Lab - www.sbglab.org Sapienza University of Rome, Italy.

\section{References}

[1] Landau L, Lifschitz EM (1980) Course of Theoretical Physics: statistical physics 5, 3rd edn. Elsevier

[2] Bizzarri M, Cucina A, Conti F, D'Anselmi F (2008) Beyond the oncogene paradigm: understanding complexity in carcinogenesis. Acta Biotheor 56(3):173-196

[3] Huang S, Ingber DE (2006) A non-genetic basis for cancer progression and metastasis: self-organizing attractors in cell regulatory networks. Breast Dis 26(1):27-54

[4] Tam WL, Weinberg RA (2013) The epigenetics of epithelial-mesenchymal plasticity in cancer. Nat Med 19(11):1438-1449

[5] Waddington CH (1957) The strategy of the genes. George Allen \& Unwin. Ltd.

[6] Chicone C (2006) Ordinary differential equations with applications, 2nd edn. Texts in Applied Mathematics 34. Springer, New York

[7] Paldi A (2012) What makes the cell differentiate. Prog Biophys Mol Biol 110(1):41-43

[8] Giuliani A (2010) Collective motions and specific effectors: a statistical mechanics perspective on biological regulation. BMC Genomics 11 (Suppl.1) S2

[9] Bravi B, Longo G (2015) The unconventionality of Nature: biology, from noise to functional randomness. In: Calude C, Dinneen M (eds) 
Unconventional Computation and Natural Computation. UCNC 2015, 3-34. Lecture Notes in Computer Science 9252, Springer

[10] Heylighen F (2001) The science of self-organization and adaptivity. The Encyclopedia of Life Support Systems, EOLSS 5(3):253-280

[11] Coleman P (2007) Frontier at your figertips. Nature 446:379

[12] Laughlin RB, Pines D, Schmalian J, Stojkovic BP, Wolynes P (2000) The middle way. Proc Nat Acad Sci USA 97(1):32-37

[13] Bizzarri M, Giuliani A, Cucina A, D'Anselmi F, Soto AM, Sonnenschein C (2011) Fractal analysis in a systems biology approach to cancer. Semin Cancer Biol 21(3):175-182

[14] Strohman RC (2000) Organization becomes cause in the matter. Nat Biotechnol 18:575-576

[15] Green S, Batterman R (2017) Biology meets Physics: reductionism and multi-scale modeling of morphogenesis. Stud Hist Philos Biomed Sci 61:20-34

[16] Ma S (1976) Modern theory of critical phenomena. Advanced Book Program

[17] Davies PC, Demetrius L, Tuszynki JA (2011) Cancer as dynamical phase transition. Theor Biol Med Model 8:1-30

[18] Bak P (1996) How Nature works. Spinger-Verlag, New York

[19] Bissell MJ, Inman J (2008) Reprogramming stem cells is a microenvironmental task. Proc Nat Acad Sci USA 105(41):15637-15638

[20] Masiello MG, Cucina A, Proietti S, Palombo A, Coluccia P, D'Anselmi F, Dinicola S, Pasqualato A, Morini V, Bizzarri M (2014) Phenotypic switch induced by simulated microgravity on MDA-MB-231 breast cancer cells. Biomed Res Int 2014, ID652434

[21] Soto AM, Maffini MV, Sonnenschein C (2008) Neoplasia as development gone awry: the role of endocrine disruptors. Internat $\mathrm{J}$ Andrology $31(2): 288-293$

[22] Arnold VI (1986) Catastrophe theory, 2nd edn. Springer Verlag 
[23] Gladwell M (2000) The tipping point: how little things can make a big difference. Little, Brown and Company

[24] Mojtahedi M, Skupin A, Zhou J, Castaño IG, Leong-Quong RYY, Chang H, Trachana K, Giuliani A, Huang S (2016) Cell fate decision as high-dimensional critical state. PLOS Biol 14(12):e2000640

[25] Thiery JP, Sleeman JP (2006) Complex networks orchestrate epithelialmesenchymal transitions. Nat Rev Mol Cell Biol 7(2):131-142

[26] Yao D, Dai C, Peng S (2011) Mechanism of the Mesenchymal-Epithelial Transition and its Relationship with metastatic tumor formation. Mol Cancer Res 9(12):1608-20

[27] Esteban MA, Bao X, Zhuang Q, Zhou T, Qin B, Pei D (2012) The mesenchymal-to-epithelial transition in somatic cell reprogramming. Curr Opin Genet Dev 22(5):423-428

[28] Quarteroni A (2009) Mathematical models in science and engineering. Notices Amer Math Soc 56(1):10-19

[29] Hwu WW (2011) GPU computing gems. Emerald \& Jade Editions, Applications of GPU Computing Series. Morgan Kaufmann Publishers, Elsevier

[30] Xu J, Lamouille S, Derynck R (2009) TGF-beta-induced epithelial to mesenchymal transition. Cell Res 19(2):156-172

[31] Barriere G, Fici P, Gallerani G, Fabbri F, Rigaud M (2015) Epithelial Mesenchymal Transition: a double-edged sword. Clin Transl Med $4(14): 1-6$

[32] Dinicola S, Fabrizi G, Masiello MG, Proietti S, Palombo A, Minini M, Harrath AH, Alwasel SH, Ricci G, Catizone A, Cucina A, Bizzarri M (2016) Inositol induces mesenchymal-epithelial reversion in breast cancer cells through cytoskeleton rearrangement. Exp Cell Res 345(1):3750

[33] Bizzarri M, Fuso A, Dinicola S, Cucina A, Bevilacqua A (2016) Pharmacodynamics and pharmacokinetics of inositol(s) in health and disease. Expert Opin Drug Metab Toxicol 12(10):1181-1196 
[34] Anderson PW (1972) More is different. Nature 177(4047):393-396

[35] Sarrió D, Rodriguez-Pinilla SM, Hardisson D, Cano A, MorenoBueno G, Palacios J (2008) Epithelial-mesenchymal transition in breast cancer related to the basal-like phenotype. Cancer Res 68(4):989-997

[36] Steinberg MS (1986) Cell surfaces in development and cancer. Springer

[37] Peinado H, Olmeda D, Cano A (2007) Snail, Zeb and bHLH factors in tumour progression: an alliance against the epithelial phenotype? Nature Reviews Cancer 7(6):415-428

[38] Maeda M, Johnson KR, Wheelock MJ (2005) Cadherin switching essential for behavioral NOT morphological changes during an epithelium to mesenchyme transition. J Cell Sci 118(Pt 5):873-887

[39] Pasqualato A, Palombo A, Cucina A, Mariggiò MA, Galli L, Passaro D, Dinicola S, Proietti S, D'Anselmi F, Coluccia P, Bizzarri M (2012) Quantitative shape analysis of chemoresistant colon cancer cells: correlation between morphotype and phenotype. Exp Cell Res 318(7):835846

[40] Chaitin GI (1974) Information-theoretic computational complexity. IEEE Trans Inform Theory IT-20(1):10-15

[41] Hoppe PS, Schwarzfischer M, Loeffler D (2016) Early myeloid lineage choice is not initiated by random PU.1 to GATA1 protein ratios. Nature 535(7611):299-302

[42] Weichsel J, Herold N, Lehmann MJ, Kräusslich HG, Schwarz US (2010) A quantitative measure for alterations in the actin cytoskeleton investigated with automated high-throughput microscopy. Cytometry A. $77(1): 52-63$

[43] Tojkander S, Gateva G, Lappalainen P (2012) Actin stress fibers assembly, dynamics and biological roles. J Cell Science 125(8):18551864

[44] Oksendal B (2000) Stochastic differential equations. Springer-Verlag

[45] Quarteroni A, Sacco R, Saleri F (2007) Numerical mathematics, 2nd edn. Texts in Applied Mathematics 37, Springer-Verlag, Berlin 
[46] O’Malley RE (1991) Singular perturbation methods for ordinary differential equations. Applied Mathematical Sciences, 89. Springer-Verlag, New York

[47] Zmeskal O, Dzik P, Vesely M (2013) Entropy of fractal systems. Comp Math Appl 66(2):135-146

[48] Spillman WB, Robertson JL, Huckle WR, Govindan BS, Meissner KE (2004) Complexity, fractals, disease time, and cancer. Phys Rev E 70:061911

[49] Chen Y (2016) Equivalent relation between normalized spatial entropy and fractal dimension. Available via arXiv.org $>$ physics $>$ arXiv:1608.02054. http://arxiv.org/abs/1608.02054 Accessed 4 May 2017

[50] Dafermos C (2005) Hyperbolic conervation laws in continuum physics, 2nd edn. Springer-Verlag

[51] Vrabie II (2004) Differential equations. An introduction to basic concepts, results and applications. World Scientific Publishing Co., Inc., River Edge, NJ

[52] Richtmyer RD, Morton KW (1994) Difference methods for initial-value problems, 2nd edn. Robert E. Krieger Publishing Co. Inc., Florida

[53] LeVeque RJ (2007) Finite difference methods for ordinary and partial differential equations. Steady-state and time-dependent problems. Society for Industrial and Applied Mathematics (SIAM), Philadelphia

[54] Sanders J, Kandrot E (2010) CUDA by example: an introduction to general-purpose GPU programming, NVIDIA Corporation, AddisonWesley

[55] Karniadakis GE, Kirby ll RM (2003) Parallel Scientific Computing in $\mathrm{C}++$ and MPI: a seamless approach to parallel algorithms and their implementation. Cambridge University Press, Cambridge

[56] Murray L (2012) GPU acceleration of Runge-Kutta integrators. IEEE Trans. Parallel Distributed Syst. 23:94-101

[57] Gantmacher F (1959) Applications of the theory of matrices. Interscience, New York 